\title{
DISTANCE GEOMETRY NOTES
}

\section{M. BLUMENTHAL}

Introduction. This paper is concerned with several disconnected developments in distance geometry. $\$ 1$ deals with the congruent imbedding of metric spaces in euclidean or Hilbert spaces. By showing that the validity of the pythagorean theorem insures the essentially euclidean character of the metric, the basic role this theorem plays in euclidean geometry is seen to be fully justified. In $\$ 2$ the circle and $n$-dimensional sphere are considered with respect to the property of covering euclidean subsets. The concluding section presents an algebraic-geometric proof of the quasi congruence order property of the $E_{n}$ which, by making use of determinants, achieves a considerable abbreviation of the two proofs of this important result hitherto published. The desired purely algebraic proof has not yet been obtained.

1. Metric spaces and the theorem of Pythagoras. If a metric space contains a line $L$ (that is, a set congruent with the euclidean straight line) and a point $p$ not on $L$, then the line contains a point $p_{0}$ nearest $p$. The pythagorean theorem is valid in the space provided for each element $x$ of $L, p p_{0}^{2}+p_{0} x^{2}=p x^{2}$. It is clear that this property insures the uniqueness of the point $p_{0}$.

THEOREM 1.1. A separable, complete, convex, externally convex metric space in which the theorem of Pythagoras is valid is congruent with a euclidean or Hilbert space.

PROOF. From an early result of the writer it suffices to show that the space has the weak euclidean four-point property; that is, each quadruple of points containing a linear triple is congruently contained in the plane. ${ }^{1}$ If $p, q, r, s$ are four such points, $L$ a line containing $q, r, s$, and $p_{0}$ the point of $L$ nearest $p$, let $q^{\prime}, r^{\prime}, s^{\prime}, p_{0}^{\prime}$ be points of an $E_{1}$ congruent with the set $q, r, s, p_{0}$. In the plane $E_{2}$ formed by this $E_{1}$ and the line perpendicular to it at $p_{0}^{\prime}$, let $p^{\prime}$ denote a point on this perpendicular with $p^{\prime} p_{0}^{\prime}=p p_{0}$. Since the theorem of Pythagoras is valid in both the given space and in $E_{2}$, it is clear that the distances of $p^{\prime}$ from the points $q^{\prime}, r^{\prime}, s^{\prime}$ equal, respectively, the distances of $p$ from $q, r, s$, and the theorem is proved.

Presented in part to the Society, September 13, 1943, under the title Nerw formulations of some imbedding theorems; received by the editors December 17, 1943.

${ }^{1}$ See Amer. J. Math. vol. 57 (1935) pp. 51-61; in particular, p. 61. 
It is worth remarking that the proof of the theorem, trivial when based upon the weak euclidean four-point property, is by no means immediate in terms of the ordinary four-point property which necessitates showing that every quadruple is congruently contained in $E_{3}$. It serves, therefore, as a good example of the usefulness of the weaker property.

If any condition leading to finite dimensionality is adjoined to those in the theorem (for example the absence of equilateral $(n+2)$-tuples or the compactness of bounded sets) the condition of separability may be suppressed (completeness also in the second alternative, of course) and congruence with a euclidean space results.

2. Covering indices. A given space is said to have congruence indices $(n, k)$ with respect to a prescribed class of spaces provided any space of the class, containing more than $n+k$ points, is congruently contained in the given space whenever each of its $n$-tuples has this property. The indices $(n, k)$ are "best" in case neither can be reduced. ${ }^{2}$

If the given space is a subset of a euclidean space and the spaces of the prescribed class are also euclidean subsets (though not necessarily of the same space of which the given space is a subset) the possession of congruence indices $(n, k)$ means that any member of the class, containing more than $n+k$ points, can be covered by the given space whenever each $n$ of its points is coverable by the space. This suggests the term "covering indices" as a specialization of the more general concept. Thus, for example, a circular disc has covering indices $(3,0)$ with respect to the class of subsets of the plane, since any plane set may be covered by such a disc whenever each three of its points are so coverable. Denoting by $C_{n, r}$ the surface of the $n$-dimensional sphere of radius $r$, with euclidean (chord) metric, we have:

LEMMA 2.1. If each three of four distinct coplanar points are coverable by the circle $C_{1, r}$, but the four points are not so coverable, then they form an orthocentric quadruple.

Proof. Let $p_{1}, p_{2}, p_{3}, p_{4}$ denote the four points, and $q_{i}$ the circumcenter of the triple obtained by omitting the $i$ th point $(i=1,2,3,4)$ (clearly no three of the points are on a line). It follows at once from the hypotheses that $q_{1}, q_{2}, q_{3}$ are reflections of $q_{4}$ in the sides $p_{2} p_{3}, p_{1} p_{3}, p_{1} p_{2}$, respectively, of the triangle $p_{1} p_{2} p_{3}$, and $p_{4} q_{1}=p_{4} q_{2}$ $=p_{4} q_{3}=r$. Thus $p_{4}$ is the isogonal conjugate of the circumcenter $q_{4}$

2 The concept of congruence indices was introduced in Bull. Amer. Math. Soc. vol. 47 (1941) pp. 435-443. 
of triangle $p_{1} p_{2} p_{3}$ and hence is the orthocenter of the triangle.

If four points form an orthocentric quadruple, the four triples have circumcircles of the same radius. But the four points are surely not concyclic, for since $p_{4}$, for example, is the isogonal conjugate of the circumcenter $q_{4}$ of the triangle $p_{1} p_{2} p_{3}$, it follows that $q_{4}$ is the isogonal conjugate of $p_{4}$ which cannot then lie on the circumcircle of $p_{1} p_{2} p_{3}$ since no point on this circle has an isogonal conjugate with respect to $p_{1} p_{2} p_{3}$. Hence a necessary and sufficient condition that four distinct coplanar points be not coverable by $C_{1, r}$, though each triple of the points be coverable, is that the four points form an orthocentric quadruple.

THEOREM 2.1. The $C_{1, r}$ has best covering indices $(3,1)$ with respect to subsets of the plane.

Proof. To prove the validity of indices $(3,1)$ it suffices to show that any planar quintuple is coverable by $C_{1, r}$ whenever each three of its points are. If, now, $p_{1}, \cdots, p_{5}$ be such a quintuple, an assumption that it is not coverable by $C_{1, r}$ implies that at least two of its quadruples be not coverable. According to the foregoing, each of these quadruples forms an orthocentric set, and since they have a triple in common, the fourth points in each quadruple coincide, for each is the orthocenter of the common triple.

That the indices $(3,1)$ are best is obvious since it is trivial to observe that the first index cannot be reduced, while the example of an orthocentric quadruple shows that the second index cannot be replaced by zero.

LEMMA 2.2. If four noncoplanar points have each triple coverable by $C_{1, r}$, then they are the vertices of an isosceles tetrahedron.

Proof. Let $O$ denote the center of the sphere circumscribing the four points and $R$ its radius. The feet of the perpendiculars from $O$ to the faces of the tetrahedron are the circumcenters of these faces and so the distances of $O$ from each face is $\left(R^{2}-r^{2}\right)^{1 / 2}$. Thus $O$ is also the center of the inscribed sphere and the assertion of the lemma follows.

LemMa 2.3. The $C_{1, r}$ has best covering indices $(3,1)$ with respect to subsets of $E_{\mathbf{3}}$.

Proof. Let $S$ be any subset of $E_{3}$ containing more than four points, each three of which are coverable by $C_{1, r}$, and let $p_{1}, \cdots, p_{5}$ be any five points of $S$. If these five points are not coverable by $C_{1, r}$ then, by Theorem 2.1, the five points are not coplanar and hence they contain at most one planar quadruple. Examining the (at least) four nonplanar quadruples in the light of Lemma 2.2, one sees that all the 
ten distances determined by the five points are equal. Since the $E_{3}$ contains no equilateral five-point, this is impossible and the lemma follows. (Since the indices $(3,1)$ are best with respect to subsets of $E_{2}$ they are a fortiori best for subsets of $E_{3}$.)

It is noteworthy that $C_{1, r}$ has the same set of best indices with respect to subsets of $E_{2}$ as it has with respect to subsets of $E_{3}$.

THeOREM 2.2. The $C_{1, r}$ has best covering indices $(3, n-2)$ with respect to subsets of $E_{n}, n>2$.

PROOF. This has been proved for $n=3$. We make the inductive assumption of the theorem's validity in $E_{n}, n>2$. Let $p_{1}, p_{2}, \cdots, p_{n+3}$ be any $(n+3)$-tuple of a subset $S$ of $E_{n+1}$ containing more than $n+2$ points, with each triple coverable by $C_{1, r}$. If this $(n+3)$-tuple be supposed not coverable by $C_{1, r}$ it follows (by the inductive hypothesis) that the $(n+3)$-tuple is not contained in $E_{n}$.

Case 1. No $(n+2)$-tuple of $p_{1}, p_{2}, \cdots, p_{n+3}$ is in $E_{n}$.

Then no quadruple of these points is in $E_{2}$ and hence (Lemma 2.2) for each quadruple $p_{i}, p_{j}, p_{k}, p_{l}$, "opposite" distances are equal. Then the $n+3$ points form an equilateral set, which is impossible since they are contained in $E_{n+1}$.

Case 2. At least one $(n+2)$-tuple of $p_{1}, p_{2}, \cdots, p_{n+3}$ is contained in $E_{n}$.

Since the $(n+3)$-tuple is not contained in $E_{n}$, it follows that in this case exactly one $(n+2)$-tuple is contained in $E_{n}$, say $p_{2}, \cdots, p_{n+3}$. By the inductive hypothesis these $n+2$ points are coverable by $C_{1, r}$, and hence they lie in a plane. But then the $n+3$ points lie in $E_{3}$, which (since $n>2$ ) contradicts the conclusion reached above that $p_{1}, p_{2}, \cdots, p_{n+3}$ are not contained in $E_{n}$.

Hence the $C_{1, r}$ has covering indices $(3, n-2)$ with respect to subsets of $E_{n}, n>2$. The presence in $E_{n}$ of equilateral $(n+1)$-tuples with each three points coverable by $C_{1, r}$ shows that the indices $(3, n-2)$ are best.

THEOREM 2.3. The $C_{n, r}$ has covering indices $(n+2,1)$ with respect to subsets of $E_{n+1}$.

Proof. Let $S$ be a subset of $E_{n+1}$ containing more than $n+3$ points and consider the semimetric space $S^{\prime}+\left(a^{\prime}\right)$, with $S^{\prime}$ congruent with $S$ and $a^{\prime} p^{\prime}=r$ for each element $p^{\prime}$ of $S^{\prime}$.

It is seen at once that each set of $n+3$ points of this semimetric space is congruently contained in $E_{n+1}$, for if the $n+3$ points are in $S^{\prime}$ this follows from the congruence of $S^{\prime}$ with $S$, while if $p_{1}^{\prime}, p_{2}^{\prime}, \cdots, p_{n+2}^{\prime}$ are any $n+2$ points of $S^{\prime}$, the $(n+3)$-tuple 
$p_{1}^{\prime}, p_{2}^{\prime}, \cdots, p_{n+2}^{\prime}, a^{\prime}$ is congruent with $p_{1}, p_{2}, \cdots, p_{n+2}, a$, where the points $p_{1}, p_{2}, \cdots, p_{n+2}$ are selected from $S$ and the point $a$ is the center of the $C_{n, r}$ passing through them.

Since $S^{\prime}+\left(a^{\prime}\right)$ contains more than $n+4$ points, and $E_{n+1}$ has, by a fundamental theorem (proof of which is given in §3), congruence indices $(n+3,1)$ with respect to the class of all semimetric spaces, it follows that this semimetric space is congruently contained in $E_{n+1}$, and as each point of $S^{\prime}$ has distance $r$ from $a^{\prime}$, it is coverable by $C_{n, r}$ and the theorem is proved.

It seems quite likely that the indices $(n+2,1)$ are best, though this has not as yet been completely established except for $n=1,2$.

It is expected that these covering properties will lead to new characterizations of the figures concerned. It has already been shown, for example, that the circular disc is characterized among all connected, simply connected domains of the plane by the property of possessing covering indices $(3,0)$ with respect to plane sets. In an analogous manner, the circular rim $C_{1, r}$ might be characterized among all simple closed curves of the plane by possessing best covering indices $(3,1)$ with respect to planar subsets (or, perhaps, among all Jordan curves of $E_{n}$ by having as best indices $\left.(3, n-2)\right) .^{3}$

3. Congruence indices of the $E_{n}$ with respect to semimetric spaces. It was shown by Menger that any semimetric space of more than $n+3$ points is congruently contained in $E_{n}$ whenever each $n+2$ of its points has this property. Thus $E_{n}$ has congruence indices $(n+2,1)$ with respect to the class of semimetric spaces or, in the older terminology, quasi congruence order $n+2$. This theorem is fundamental in the metric study of euclidean space.

The literature contains only two proofs of this important result, both of which are quite lengthy and necessitate considerable indoctrination of the reader in the preceding theory. ${ }^{4}$ This is due, in part, to the fact that the argument is entirely geometrical and demonstrates more than is actually needed in order to arrive at the desired result. Since the theorem is easily formulated in the language of determinant or quadratic form theory, attempts have been made to furnish a completely algebraic proof of the theorem. The develop-

8 In this connection it would be of interest to prove or disprove the writer's conjecture that corresponding to each plane simple closed curve there exists a circle with each three of its points coverable by the simple closed curve. In the event that the curve is a triangle, the inscribed circle serves as the circle of the conjecture.

4 The proofs are by Karl Menger, Math. Ann. vol. 100 (1928) pp. 120-130 and Amer. J. Math. vol. 53 (1931) pp. 730-737. 
ments presented here do not accomplish this but they do employ determinant theory to considerably shorten the proofs heretofore obtained, and they hew close to the line by proving only those facts which are needed to establish the result. Thus the end is achieved more speedily though the route travelled is not essentially different. ${ }^{5}$

Calling a set pseudo- $E_{n}$ provided it is not congruently contained in $E_{n}$ though each of its $(n+2)$-tuples is, we show first the following lemma.]

Lemma 3.1. $A$ pseudo- $E_{n}(n+4)$-tuple $P$ contains at least $n+2$ pseudo- $E_{n}(n+3)$-tuples.

Proof. Suppose this is not the case. Then $P$ contains at least three $(n+3)$-tuples which are congruently contained in $E_{n}$. Now since any three $(n+3)$-tuples of $P$ have $n+1$ points in common, we may assume the labelling so that $p_{1}, p_{2}, \cdots, p_{n+1}, p_{n+2}, p_{n+3}$; $p_{1}, p_{2}, \cdots, p_{n+1}, p_{n+2}, p_{n+4} ; p_{1}, p_{2}, \cdots, p_{n+1}, p_{n+8}, p_{n+4}$ are euclidean $(n+3)$-tuples. Then the determinant $D\left(p_{1}, p_{2}, \cdots, p_{n+8}, p_{n+4}\right)$ of the set $P$ has rank less than or equal to $n+2$, since the principal minors $D\left(p_{1}, p_{2}, \cdots, p_{n+1}, p_{n+i}\right)$ vanish for $i=2,3,4$ (every $n+2$ points of $P$ being euclidean) and

$$
\begin{aligned}
D\left(p_{1}, \cdots, p_{n+1}, p_{n+2}, p_{n+3}\right) & =D\left(p_{1}, \cdots, p_{n+1}, p_{n+2}, p_{n+4}\right) \\
& =D\left(p_{1}, \cdots, p_{n+1}, p_{n+3}, p_{n+4}\right)=0
\end{aligned}
$$

(since the three $(n+3)$-tuples are euclidean).

It follows that each $(n+3)$-tuple of $P$ has a vanishing determinant $D$ and hence is congruently contained in $E_{n}$. But this implies that $P$ is euclidean, contrary to the hypothesis.

Lemma 3.2. Let $P$ be a pseudo- $E_{n}(n+3)$-tuple. Then each $n+2$ points of $P$ contains an independent $(n+1)$-tuple.

Proof. If this is not the case we may assume the labelling so that $p_{1}, p_{2}, \cdots, p_{n+2}$ are $n+2$ points of $P$ with each of its $(n+1)$-tuples dependent. Then $D\left(p_{1}, p_{2}, \cdots, p_{n+2}\right)$ has rank not exceeding $n+1$, for $D\left(p_{1}, p_{2}, \cdots, p_{n+2}\right)$ vanishes and $D\left(p_{1}, p_{2}, \cdots, p_{n}, p_{n+1}\right)$ $=D\left(p_{1}, p_{2}, \cdots, p_{n}, p_{n+2}\right)=D\left(p_{1}, p_{2}, \cdots, p_{n}, p_{n+1}, p_{n+2}\right)=0$. Thus all first minors of $D\left(p_{1}, p_{2}, \cdots, p_{n+2}\right)$ vanish, and hence $D\left(p_{1}, p_{2}, \cdots, p_{n+3}\right)$ is zero. This is impossible since $P$ is a pseudo- $E_{n}$ $(n+3)$-tuple.

D We are referring here to the second of the two references in the preceding footnote. 
LeMma 3.3. Let $P$ and $Q$ be two pseudo- $E_{n}(n+3)$-tuples with $n+2$ points of $P$ congruent with $n+2$ points of $Q$. Then $P$ and $Q$ are congruent.

Proof. By the preceding lemma the $n+2$ points of $P$ which are congruent to $n+2$ points of $Q$ contain an independent $(n+1)$-tuple. Selecting the labelling so that the $n+2$ points $p_{1}, p_{2}, \cdots, p_{n+1}, p_{n+2}$ of $P$ are congruent with the $n+2$ points $q_{1}, q_{2}, \cdots, q_{n+1}, q_{n+2}$ of $Q$, it follows easily that the distances of $p_{n+3}$ from the points of the first set are the same functions of the mutual distances of $p_{1}, p_{2}, \cdots, p_{n+2}$ as the distances of $q_{n+3}$ from the points of the congruent set $q_{1}, q_{2}, \cdots, q_{n+2}$, and hence the two $(n+3)$-tuples are congruent.

THEOREM 3.1. If a semimetric space $S$ of more than $n+3$ points has each of its $(n+2)$-tuples congruently contained in $E_{n}$, then $S$ is congruently contained in $E_{n}$.

Proof. Suppose $S$ is not congruently contained in $E_{n}$. Then $S$ has at least one $(n+3)$-tuple with this property, and any $(n+4)$-tuple containing this $(n+3)$-tuple is a pseudo- $E_{n}$ set. Let $P=\left(p_{1}, p_{2}, \cdots, p_{n+4}\right)$ be such an $(n+4)$-tuple. By Lemma 3.1 at most two of the $(n+3)$ tuples of $P$ can be euclidean, say $p_{1}, p_{2}, \cdots, p_{n+2}, p_{n+3}$ and $p_{1}, p_{2}, \cdots, p_{n+2}, p_{n+4}$.

To show that $p_{1}, p_{2}, \cdots, p_{n+2}$ forms an equilateral set (which furnishes the desired contradiction to the assumption that $S$ is not congruently contained in $E_{n}$ ) let $p_{i} p_{j}, p_{r} p_{s}$ be any pair of its nonzero distances. Then the indices $i, j, r, s$ are selected from the numbers $1,2, \cdots, n+2$, and so the two $(n+3)$-tuples $p_{1}, p_{2}, \cdots, p_{i-1}, p_{i+1}, \cdots, p_{r-1}, p_{r+1}, \cdots, p_{n+2}, p_{n+3}, p_{n+4}, p_{i}$; $p_{1}, p_{2}, \cdots, p_{i-1}, p_{i+1}, \cdots, p_{r-1}, p_{r+1}, \cdots, p_{n+2}, p_{n+3}, p_{n+4}, p_{r}$ are pseudo- $E_{n}$. From Lemma 3.3 we have $p_{i} p_{j}=p_{r} p_{j}$.

But from the two pseudo- $E_{n}(n+3)$-tuples $p_{1}, p_{2}, \cdots, p_{j-1}, p_{j+1}$, $\cdots, p_{s-1}, p_{s+1}, \cdots, p_{n+2}, p_{n+8}, p_{n+4}, p_{j} ; p_{1}, p_{2}, \cdots, p_{j-1}, p_{j+1}, \cdots$, $p_{s-1}, p_{s+1}, \cdots, p_{n+2}, p_{n+3}, p_{n+4}, p_{s}$ we have $p_{j} p_{r}=p_{s} p_{r}$. Hence $p_{i} p_{j}=p_{r} p_{s}$ and the $n+2$ points $p_{1}, p_{2}, \cdots, p_{n+2}$ form an equilateral $(n+2)$-tuple. They are not, then, congruently contained in $E_{n}$, contrary to the hypothesis, and the theorem is proved.

The University OF Missouri 